\title{
COMPRENSIÓN DE LOS CAMBIOS EN LA CONCEPTUALIZACIÓN DEL TRASTORNO DEL ESPECTRO AUTISTA PARA LA INCLUSIÓN EDUCATIVA
}

\author{
CARMEn SANDOVAL \\ https://orcid.org/0000-0003-3239-4158 \\ FLOR QUISPE \\ https://orcid.org/0000-0001-9988-2098 \\ ROSA GUILLÉN \\ https://orcid.org/0000-0002-7480-8008 \\ Pontificia Universidad Católica del Perú, Lima, Perú \\ Correo electrónico: sandoval.cm@pucp.pe \\ Recibido: 19 de octubre del 2021 / 3 de noviembre del 2021 \\ doi: https://doi.org/10.26439/persona2021.n024(2).5562
}

\begin{abstract}
RESUMEN. El artículo presenta los resultados de una revisión de literatura que explica los cambios en la conceptualización del trastorno del espectro autista (TEA) durante el último siglo, desde los estudios de Bleuler hasta la actualidad. La información bibliográfica fue recogida durante los meses de octubre del 2020 y abril del 2021. El análisis realizado muestra que el concepto sobre el TEA ha variado a lo largo del tiempo, pasando por diversas posturas: primero fue entendido desde un plano psiquiátrico hasta lo que ahora se reconoce como un trastorno del neurodesarrollo. Se detallan las principales características y signos de alarma que acompañan al TEA en la primera infancia, desde la perspectiva actual que lo enmarca dentro de los trastornos del neurodesarrollo y señala su carácter dimensional. La información que aporta esta investigación está orientada hacia los principales agentes involucrados en la educación de los niños diagnosticados con TEA, para que pueda ser divulgada entre los docentes y las personas interesadas a fin de poder identificar las señales de alerta antes de los tres primeros años de vida.
\end{abstract}

Palabras clave: autismo / educación de la primera infancia / primera infancia / necesidad de educación / protección a la infancia 


\title{
UNDERSTANDING THE CHANGES IN THE CONCEPTUALIZATION OF AUTISM SPECTRUM DISORDER FOR EDUCATIONAL INCLUSION
}

\begin{abstract}
The article presents the results of a literature review that explains the changes in the conceptualization of autism spectrum disorder (ASD) during the last century, from Bleuler studies to the present day. The bibliographical research was done during the months of October 2020 and April 2021. The analysis shows ASD concept has changed all over time. Initially it was understood from a psychiatric level and it is currently recognized as a neurodevelopmental disorder. This research also details the main characteristics and early warning signs of Austism Spectrum Disorder (ASD) in early childhood from the current point of view which frames it within the neurodevelopmental disorders and points out that it has a dimensional approach. The information provided by this research is oriented towards the main agents involved in the education of children diagnosed with ASD, so that it can be disclosed among teachers and interested people in order to identify the early signs and symptoms before the first three years of life.
\end{abstract}

Keywords: autism / early childhood education / early childhood / educational need / child protection 


\section{INTRODUCCIÓN}

La comprensión del trastorno del espectro autista (TEA) se ha ido construyendo desde diversas perspectivas teóricas, y cada uno de estos esfuerzos no solo ha contribuido a la evolución del concepto, sino también a la denominación asociada a esta condición y su tratamiento educativo. El conocimiento del TEA de parte de los docentes permite que enfrenten sus creencias, y rompan estereotipos y etiquetas asociadas a la condición, lo que influye de manera positiva en su práctica educativa y en generar un clima de aula óptimo entre los niños (Valdez, 2019). Actualmente, el TEA es definido como un trastorno del neurodesarrollo que acompañará a la persona a lo largo de su vida afectándola directamente y limitando las relaciones que establezca con los demás y con su entorno (Manrique et al., 2019). Respecto a su prevalencia, se puede observar que, en el año 2013, la Organización Mundial de la Salud (OMS) estimó que su frecuencia era equivalente a $62 / 10000$, lo que se traduce a que 1 de cada 160 niños estaba afectado por esta condición (OMS, 2013).

Existe una serie de barreras que pueden limitar el acceso de estos estudiantes a la escuela; estas giran en torno a aspectos personales, sociales, culturales y económicos, entre otros (Cueto et al., 2018). Las políticas que se diseñan para superar la exclusión reconocen estas problemáticas y realizan esfuerzos para abordarlas de forma integral. Por ello, en el Perú se plantea un marco normativo nacional de educación inclusiva para reconocer y respetar el acceso de los niños a la educación de calidad y orientar esfuerzos de forma satisfactoria, que contribuyan a la mejora de sus aprendizajes. A continuación, se señalan las principales normativas para la inclusión educativa en el país.

La Ley 28044, Ley General de Educación, promulgada el 28 de julio del 2003, en su artículo 8, declara la necesidad de reconocer los principios de acción del sistema educativo, entre los que se encuentran la equidad, la inclusión y la calidad, para asegurar las condiciones que permitan una educación integral, abierta, flexible y permanente. El Decreto Supremo 011-2012-ED, aprobado en el año 2012 como parte del reglamento de dicha ley, menciona que la educación es un derecho que debe permitir a los estudiantes el acceso a un sistema educativo de calidad, que asegure la permanencia en este, que los guíe para enfrentar diversos retos, ejercer la ciudadanía y continuar el aprendizaje a lo largo de la vida.

La Ley 30150, Ley de Protección de las Personas con Trastorno del Espectro Autista (TEA), decretada en el año 2014, busca reconocer el derecho de las personas con TEA y establecer la importancia del diagnóstico temprano, así como la detección oportuna, una adecuada intervención en función de las características del niño, la inclusión educativa, la capacitación de los profesionales que brindan atención y la creación de dispositivos para asegurar la inserción laboral y pertinente durante la adultez (Ministerio de la Mujer 
y Poblaciones Vulnerables, \& Consejo Nacional para la Integración de la Persona con Discapacidad, 2019)'.

Como consecuencia de estas políticas, se sustenta la necesidad de que las escuelas incorporen estas normativas y las reconozcan como aspectos fundamentales para su rumbo educativo. Entonces, no solo deben conocer la importancia de la inclusión de niños con TEA, sino también favorecer su inclusión a partir del conocimiento de esta condición. El proceso inclusivo de escolarización ha conducido a los centros educativos a regirse por uno de los modelos escolares: exclusión, segregación, integración e inclusión educativa (Cueto et al., 2018). En cuanto a la inclusión, es “la participación de todos los estudiantes y adultos. Trata de apoyar a los centros escolares para que sean más responsables ante la diversidad de su alumnado, sea en razón de sus orígenes, intereses, experiencias, conocimiento, capacidades o cualquier otra" (Booth \& Ainscow, 2000 , p. 13). Así, podemos entender que un aula inclusiva garantiza igualdad a todos los estudiantes, de manera que sean capaces de aprender, ser y hacer, eliminando todas las concepciones o prejuicios que podrían generar una barrera para el aprendizaje.

\section{METODOLOGÍA}

Se trata de una investigación bibliográfico-narrativa (Alayza, 2020). La investigación bibliográfica "tiene un carácter particular, de donde le viene su consideración interpretativa. Intenta leer y otorgar sentido a unos documentos que fueron escritos con una intención distinta a esta dentro de la cual se intenta comprenderlos" (Gómez, 2011, p. 230). Puede entenderse como un proceso que nos permite describir, analizar, comprender o comparar fuentes académicas (Ávila, 2006). Como afirma Alayza (2020), la revisión bibliográfica de la literatura trabaja fuentes secundarias de información e "incluye materiales publicados que proporcionan un estudio de la literatura reciente o actual. [...] Puede o no incluir una búsqueda exhaustiva. La síntesis es típicamente narrativa y el análisis puede ser cronológico, conceptual, temático, etc." (p. 26).

El procedimiento para recoger la información incluyó una revisión bibliográfica de la literatura sobre el tema de estudio publicada en un periodo de veinte años (después del 2000), sin excluir textos más antiguos, pero que son pilares para comprender el TEA. Se seleccionaron 42 fuentes en inglés y 30 en español considerando como criterio de inclusión temática básica la educación de personas con condición de TEA. Finalmente, se escogieron para este estudio 11 textos en inglés y 15 en español. Luego de una revisión,

1 "El incremento anual de las inscripciones de las personas con trastorno del espectro autista en el registro a cargo del CONADIS ha sido notorio en los últimos años, alcanzando a 501 en el año 2015, 588 en el año 2016, 873 en el año 2017 y 819 hasta el 31 de agosto del año 2018" (Ministerio de la Mujer y Poblaciones Vulnerables, \& Consejo Nacional para la Integración de la Persona con Discapacidad, 2019, p. 5). 
se dispuso la información en matrices de sistematización; después, se analizaron las fuentes consultadas agrupándolas en categorías y se organizaron las temáticas que se van a presentar.

El interés por este tema parte de conocer cómo ha sido la evolución de la conceptualización del TEA desde las primeras descripciones de Bleuler hasta la actualidad, y cuáles son los principales indicadores que pueden considerar los docentes para una identificación oportuna de los signos de alerta y características de este trastorno. Sobre esta base, se plantearon dos objetivos: (i) conocer la evolución del TEA desde las primeras descripciones de Bleuler hasta la actualidad; y (ii) describir los principales indicadores de alerta de este trastorno que pueden ser identificados por los docentes en la etapa de la primera infancia.

La ética en la investigación es un aspecto clave; por ello, se tomaron en cuenta los siguientes los principios del Comité de Ética de la Investigación de la Pontificia Universidad Católica del Perú (2016): justicia, integridad científica y responsabilidad al asumir la planificación y ejecución de la investigación juiciosamente.

\section{RESULTADOS}

\section{Cambios de un concepto complejo: del autismo al trastorno del espectro autista}

EI TEA ha sido objeto de debate no solo por la comprensión de su naturaleza, sino también por los esfuerzos por ser entendido desde los planos psicológicos, biológicos y terapéuticos. Con opiniones enfrentadas y entrelazadas, el concepto de autismo ha evolucionado hasta lo que actualmente se conoce como trastorno del espectro autista.

Bleuler y Bleuler (1986), en su investigación "Dementia praecox oder Gruppe der Schizophrenien", hacen referencia por primera vez al término autismo, que proviene de la etimología griega, en donde autos significa "sí mismo". Al respecto, Garrabé (2012) menciona:

El autismo está caracterizado según él [Bleuler] por el repliegue de la vida mental del sujeto sobre sí mismo, llegándose a la constitución de un mundo cerrado separado de la realidad exterior y a la dificultad extrema o la imposibilidad de comunicarse con los demás que de allí resulta. (p. 257)

Dicho de otro modo, el sujeto se aleja del plano exterior, replegándose y ensimismándose, para vivir dentro de su propio mundo. Bajo este enfoque, se entendía el autismo desde un paradigma psiquiátrico referido a la esquizofrenia o psicosis.

En la década de los cuarenta, específicamente en 1943, Kanner retoma el término de Bleuler en sus investigaciones al observar a once niños que presentaban ciertas conductas de aquello que previamente se conocía como autismo. De acuerdo con Diazgranados y 
Tebar (2019), Kanner define el autismo como "un trastorno de origen biológico, cuya alteración esencial consiste en una perturbación innata del contacto afectivo" (p. 4). Así, el niño evita relacionarse con los demás, tiene dificultades para el contacto visual y tiende a ignorar o desatender, además de evidenciar problemas en aspectos relacionados con la comunicación y el lenguaje (ecolalias, inversión deíctica, falta de intención comunicativa en el uso del lenguaje e insistencia en la invariancia). En sus investigaciones, se acuñó el nombre de autismo infantil precoz. Al respecto, Valdez (2005) señala que Kanner separa al autismo de las enfermedades psiquiátricas, y se centra en describir las dimensiones afectadas en el desarrollo infantil, tales como trastornos de la relación, trastornos de la comunicación y el lenguaje, y, por último, trastornos de la flexibilidad.

Un año después, en 1944, Asperger plantea la psicopatía autista, a través de la cual estudia casos vinculados al autismo de alto funcionamiento. Para ello, realiza observaciones a un grupo de niños con algunas características definidas por Kanner; sin embargo, los comportamientos eran diferentes. Según González (2015), Asperger describe a los niños como sujetos que presentan un lenguaje verbal desarrollado caracterizado por parecer petulante, una comunicación no verbal pobre, dificultad para relacionarse y hacer amigos, torpeza en las habilidades motoras y fijación en temas específicos. Artigas-Pallares y Paula (2012) sostienen que "Asperger solía utilizar la denominación de pequeños profesores (kleine Professoren) para referirse a ellos, destacando su capacidad para hablar de sus temas favoritos de modo sorprendentemente detallista y preciso" (p. 574). Como reconocimiento a su trabajo, se opta por denominar el autismo de alto funcionamiento como síndrome de Asperger.

Entre las décadas de los cuarenta y sesenta, desde una perspectiva psicoanalítica, se plantea que el autismo es un trastorno emocional que se soluciona a través de terapias afectivas. Bajo este enfoque, se atribuye la total responsabilidad a los padres, específicamente a la madre y el distanciamiento que ella tiene con sus hijos (la madre nevera de Bruno Bettelheim). Esta teoría fue apoyada por Kanner, pero luego él la rechaza al darse cuenta del efecto negativo que causa en las familias, al responsabilizarlas y culparlas por el trastorno que acompaña a sus hijos. Adicionalmente, en este mismo periodo, se elaboran tanto la CIE (Clasificación Internacional de Enfermedades) como el DSM (Manual diagnóstico y estadístico de los trastornos mentales).

Entre las décadas de los sesenta y ochenta, la perspectiva migró hacia un enfoque más educativo desde una visión cognitiva. En estos años, destacan las contribuciones de Wing y Gould (1979), quienes retoman las investigaciones de Hans Asperger y plantean el autismo en términos de espectro autista. Wing (1998, como se cita en Diazgranados \& Tebar, 2019) describe una tríada característica en la que se evidencia la alteración de tres áreas en el desarrollo del ser humano (véase la figura 1), todo ello asociado a la presencia de intereses restringidos y patrones repetitivos de conducta. 
Figura 1

Tríada de Wing

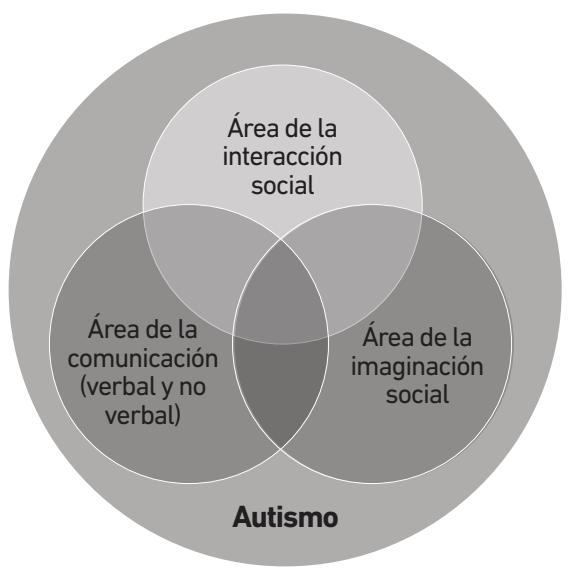

Elaboración propia

Este aporte logró que se visibilice la importancia de abordar el autismo desde los aprendizajes; por ello, se crearon centros especializados en esta área. Esto permitió que los niños con autismo desarrollen su independencia y autonomía, y estuvieran preparados para ingresar a la escolarización regular (Artigas-Pallares \& Paula, 2012). A partir de 1983, existe un predominio de los enfoques comunicativos, psicogenéticos y de relaciones interpersonales centrados en apoyar a las personas con autismo, por lo que se plantea el método de intervención ABA (Applied Behaviour Analysis).

Posteriormente, en 1994, se desarrolla el DSM-IV, que define los trastornos generalizados del desarrollo a través de un enfoque categorial y se postula un conjunto de trastornos que se asocian directamente con la tríada propuesta por Wing. Así, se describen cinco categorías: trastorno autista, síndrome de Asperger, trastorno generalizado del desarrollo no especificado, síndrome de Rett y trastorno desintegrativo infantil. Del mismo modo, se proponen tres características: los patrones de comportamiento (intereses y actividades restringidos, repetitivos y estereotipados), la alteración cualitativa de la interacción social y la alteración cualitativa de la comunicación (American Psychiatric Association, 1995).

En mayo del 2013, se publica el DSM-5, manual que se utiliza actualmente, y se observa que los cuatro trastornos descritos previamente pasan a ser observados a partir de lo que se denomina el trastorno del espectro autista. En esta agrupación se excluye el síndrome de Rett, se elimina la categoría de trastorno generalizado del desarrollo y se engloba en un continuo que depende de la afectación. También se precisa que existen diversas formas de TEA que varían en función de cada individuo y se plantean 
tres niveles de severidad (nivel 1, nivel 2 y nivel 3), divididos según las necesidades de apoyo que requiera el sujeto. Se caracteriza al TEA por (i) deficiencias persistentes en la comunicación social, y (ii) patrones restrictivos y repetitivos de comportamiento, intereses 0 actividades ${ }^{2}$.

En síntesis, aquello que se entiende por autismo hoy se ha construido a lo largo de los años gracias a las aportaciones de diversos autores e investigadores. Por este motivo, es preciso que se oriente a los profesionales a fin de que se comprenda lo que se conoce actualmente como TEA.

\section{Conceptualización del trastorno del espectro autista}

Los trastornos del neurodesarrollo (TN) son comprendidos como aquellas alteraciones o retrasos en las funciones relacionadas con la maduración del sistema nervioso, tales como las funciones motoras, cognitivas y emocionales. Estas se inician en la primera infancia y van evolucionando y presentándose en diferentes etapas de la vida del individuo. Reynoso et al. (2015) entienden el TEA como un trastorno del neurodesarrollo que afecta tanto la parte emocional como la contención de las conductas repetitivas. Esto tiene incidencia biológica; por lo tanto, se debe estar atento a los síntomas que aparecen a partir de los dieciocho meses.

Al respecto, González (2015) plantea el TEA como un síndrome que se distingue por un conjunto de síntomas que aparecen en los primeros tres años de edad y señala que esta condición acompañará al sujeto a lo largo de su vida. Con la información recopilada y presentada anteriormente, se comprende al TEA como una condición de afectación del neurodesarrollo que determina la forma en la que el niño percibe el mundo y las relaciones con los demás. Se caracteriza por una comunicación social deficiente, así como por patrones repetitivos y estereotipados del comportamiento. Se origina en los primeros tres años de edad, específicamente entre los dieciocho y treinta y seis meses, por lo que resulta imprescindible prestar atención a las señales de alerta, tanto en contextos familiares como escolares, de tal forma que se pueda detectar, derivar e intervenir, aplicando

2 Luego de que un niño es diagnosticado con TEA, se busca brindarle atención oportuna con el propósito de continuar con su proceso de desarrollo integral. En el DSM-5, se establecieron tres niveles de severidad que giran en torno a cuánta ayuda se le debe proporcionar al niño para fortalecer tanto sus capacidades comunicativas y adaptativas, como su capacidad para expandir sus intereses restringidos y repetitivos (American Psychiatric Association, 2013). La importancia de reconocer estos niveles radica en que facilita el diagnóstico por parte de los especialistas y constituye una orientación a los docentes para comprender las principales fortalezas y limitaciones que puede tener su estudiante. De esa manera, estos podrán ofrecer oportunas estrategias de aprendizaje para sus principales necesidades. Usualmente, el TEA está acompañado de una comorbilidad. Según Morueco Alonso (2015), el 50 \% de los niños diagnosticados con TEA presenta discapacidad intelectual y alrededor de un 50 \% tiene dificultades para el desarrollo del lenguaje oral; esto puede influir en los niveles de severidad del TEA en cada niño. 
estrategias educativas y creando contextos amigables para garantizar la inclusión en la escuela (Valdez, 2019).

\section{Características del trastorno del espectro autista}

Actualmente, el TEA se caracteriza principalmente por (i) deficiencias en la comunicación e interacción social que se pueden visualizar en distintos contextos; y (b) por patrones de comportamiento, intereses y actividades restringidos y repetitivos. Cada uno de estos comprende una serie de criterios que mejoran la comprensión de esta condición, los cuales se describen a continuación.

\section{Deficiencias en la comunicación e interacción social}

Se sabe que la comunicación y la interacción social son aspectos fundamentales para el desarrollo integral del niño. Estas no solo le permiten comprender el mundo que le rodea, sino también aprender diversas formas de estar y ser en el mundo, de jugar, relacionarse con otros e interactuar, de expresar sus sentimientos, ideas o necesidades. Por tanto, es preciso vislumbrar cuáles son esas características que se pueden observar en un niño que presenta deficiencias en este aspecto.

\section{Déficit en la reciprocidad socioemocional}

El ser humano tiene la capacidad de relacionarse con los demás y, fruto de estas relaciones sociales y emocionales, adquiere la capacidad para entender y responder ante las emociones externas. Según Miguel (2006), estas se originan en los primeros años de vida cuando el niño desarrolla la habilidad para responder ante las señales de su cuidador principal. Este autor señala que los niños con TEA nacen incapaces de establecer relaciones con su cuidador. Por tanto, no podrán comprender, interpretar ni responder ante las emociones de los demás.

Una característica clave en los primeros meses de vida es el desarrollo de la atención conjunta, porque es el principal signo de comunicación que se establece entre el cuidador y el bebé aproximadamente a los nueve meses. A medida que el bebé va creciendo, pasa de una comunicación asimétrica, en donde el adulto tiene un rol protagonista, a una comunicación intencionada en donde existe reciprocidad entre ambos interlocutores (bebé-cuidador).

Otro aspecto relacionado con la comunicación durante los primeros meses es la sonrisa social, mediante la cual el niño establece lazos comunicativos con su cuidador e interactúa ante las señales y mensajes que se le envía. La sonrisa social permite no solo la comunicación, sino también el establecimiento de lazos afectivos entre ambos. En los casos de niños con TEA, este aspecto se visibiliza en las primeras interacciones, cuando el cuidador envía un mensaje y no obtiene respuesta por parte del menor. Busquets et al. 
(2018) la describen como una sonrisa fría, carente de significado que no responde a la interacción ni envía una respuesta.

También se pueden identificar problemas relacionados con la empatía. Los niños diagnosticados con TEA presentan dificultades para reconocer las emociones simples y complejas. Peretti (2010) plantea que a los cinco o seis meses de edad los niños tienen la capacidad para distinguir las emociones de los demás. La comprensión de las emociones complejas puede provocar estados emocionales en las personas, ya que son fruto de las expectativas y creencias de los individuos. Este entendimiento se logra a los cinco o seis años cuando comprenden que pueden causar un efecto directo en las emociones de las personas. Los niños con TEA precisan de una mayor cantidad de tiempo para entender las emociones. Este problema se puede observar cuando los niños envían mensajes poco empáticos al otro, pese a que se observa una emoción clara en el receptor, o cuando no comprenden que las personas pueden tener pensamientos o creencias diferentes de los suyos.

\section{Déficit en conductas comunicativas no verbales utilizadas en la interacción social}

Algunas de las conductas comunicativas no verbales son las protoimperativas y protodeclarativas, que constituyen un hito significativo en el desarrollo comunicativo (Valdez, 2012). Ambas son consideradas la materialización de un proceso de triangulación, conocido como esquema triádico único, en el cual el niño de forma intencional es capaz de coordinar e interactuar con los objetos y las personas.

En el TEA, las conductas protoimperativas se encuentran afectadas, puesto que los niños muestran ausencia de intención para pedir o solicitar algo haciendo uso de gestos o medios no lingüísticos. Asimismo, al verse afectada la conducta protoimperativa, incide en el desarrollo de la conducta protodeclarativa, observándose que los niños poseen deficiencias para compartir eventos, objetos o acciones de su interés. En ese sentido, Bruinsma et al. (2004) indican:

[...] children with autism appear to produce and understand protoimperative pointing to some extent so that this is a relative strength, but protodeclarative pointing is severely im-paired, and often completely absent. (p. 171) $)^{3}$

En otras palabras, las conductas protoimperativas pueden llegar a desarrollarse en estos niños; sin embargo, en ocasiones, pueden estar carentes de intención social. Por otro lado, las conductas protodeclarativas suelen ser las más difíciles de lograr, por lo que a menudo están ausentes al tener limitaciones para compartir la experiencia.

3 “Los niños con autismo parecen producir y comprender el señalar protoimperativo hasta cierto punto, por lo que esto es una fortaleza relativa, pero el señalar protodeclarativo está gravemente afectado y, a menudo, está completamente ausente" [traducción de las autoras]. 
Es importante resaltar la mirada conjunta como un signo de socialización entre el niño y el adulto. En el TEA, el niño no fija la mirada en los ojos de su cuidador y parece no tener interés en él. Al respecto, Wang et al. (2014) resaltan que "people with autism not making eye contact suffer from many social issues including an inability to communicate effectively and solitude" (p. 2) 4 . En ese sentido, es necesario que se desarrollen estrategias clave para favorecer este aspecto, ya que el contacto visual es un proceso íntimo y complejo en el cual se envía el mensaje al receptor de que se le está atendiendo en todo el sentido de la palabra.

\section{Déficit en el desarrollo y mantenimiento de las relaciones sociales}

Las principales dificultades dentro de este déficit se centran en las características del juego, el juego simbólico y la teoría de la mente. El juego cumple un papel sumamente importante en el desarrollo integral del niño, debido a que favorece su aprendizaje; estructura su personalidad; estimula habilidades creativas, sensoriales y físicas; favorece los procesos cognitivos básicos como la atención, percepción y memoria; y contribuye a su desarrollo emocional y social (Meneses Montero \& Monge Alvarado, 2001).

En el caso de los niños con TEA, se observa que está afectado notoriamente el aspecto simbólico, el cual representa el acto en el cual el niño le otorga un significado diferente al objeto o juguete con el que se relaciona; por ejemplo, el niño utiliza una caja simulando que es una nave espacial (Piaget, 1991). En ese sentido, se propone la siguiente tipología: (i) sustitución de objetos, objeto que toma la representación de otro objeto; (ii) atribución de características falsas, atribuir propiedades a los objetos como si fueran reales; y (iii) simulación de un objeto ausente, referirse a un objeto ausente como si estuviera presente.

Lam (2014), a partir de diversos aportes científicos relacionados con el juego simbólico y niños con autismo, sostiene lo siguiente:

[...] children with autism, particularly those older and high-functioning children, are able to engage in symbolic play when cues or prompts were provided but not when they were expected to produce pretense spontaneously [...] some studies had made it clear that even under structured conditions and with prompts provided, children with autism still displayed deficits in symbolic play. (párr. 6) ${ }^{5}$

4 “Las personas con autismo que no hacen contacto visual sufren muchos problemas sociales, incluida la incapacidad para comunicarse de manera efectiva y la soledad" [traducción de las autoras].

5 "Los niños con autismo, en particular los niños mayores y de alto funcionamiento, son capaces de participar en juegos simbólicos cuando se les proporcionaron señales o indicaciones, pero no cuando se esperaba que produjeran una simulación de forma espontánea [...] algunos estudios habían dejado en claro que incluso en condiciones estructuradas y con indicaciones proporcionadas, los niños con autismo aún mostraban deficiencias en el juego simbólico" [traducción de las autoras]. 
Estas deficiencias presentes en los niños diagnosticados con TEA pueden ser explicadas a partir de la teoría de la mente (ToM), la teoría de la disfunción ejecutiva y la teoría de la coherencia central. La primera sostiene que existe un déficit en la metarrepresentación, que es la capacidad para asignarle una nueva identidad a un objeto. La segunda indica una alteración en las funciones ejecutivas causada por la incapacidad del sujeto para abstraerse de la realidad, iniciar un juego simbólico y cambiar de identidad de una primaria a una imaginaria. La última refiere a un deterioro en la coherencia central, en el cual se perciben dificultades para comprender la simulación, para su ejecución y producción (Lam, 2014).

Por otro lado, los aspectos notorios en la forma de jugar de estos niños son, primero, la tendencia a centrarse en los detalles de los juguetes (color, características, entre otros) y, segundo, las características repetitivas y restringidas del juego. En estos niños se presenta con mayor incidencia la presencia del juego funcional, centrado únicamente en las funcionalidades del objeto con el que se relaciona.

\section{Patrones restrictivos y repetitivos de comportamiento}

EI TEA se caracteriza por la presencia de patrones restrictivos y repetitivos del comportamiento; por ello, para una mejor comprensión, se explican detalladamente los aspectos que lo componen.

Lenguaje, movimientos, utilización de objetos estereotipados y repetitivos

Las ecolalias, las estereotipias motoras y el uso de objetos de forma repetitiva y constante son características que se pueden observar en esta sección. Las primeras se desprenden del lenguaje y consisten principalmente en la repetición de mensajes que se han escuchado previamente; estas pueden desaparecer a medida que se va desarrollando el habla en los niños. Empleando las palabras de Mergl y Salgado (2015), "repetitive behaviors in language can be manifested by the occurrence of echolalia, persistent phenomenon characterized as a language impairment, with repetition of the speech of the other, divided into immediate or delayed" (p. 2072).

Según Mergl y Salgado (2015), esta repetición de palabras es usada como dispositivo de comunicación, confirmación de deseos, regulación de conductas o medios para entablar una conversación cuando aún tienen dificultades para usar sus propias palabras. Así, deben ser vistas como medio de interacción y comunicación, y no solo como un problema patológico.

6 “Las conductas repetitivas en el lenguaje pueden manifestarse por la aparición de ecolalia, fenómeno persistente caracterizado como una alteración del lenguaje, con repetición del habla del otro, dividido en inmediato o retardado" [traducción de las autoras]. 
En cuanto a las alteraciones del movimiento, se pueden localizar las estereotipias motoras. Harris (2008, como se cita en Péter et al., 2017) señala que estas son "repetitive, rhythmic, often bilateral movements with a fixed pattern (e. g., hand flapping, waving, or rotating) and regular frequency that can usually be stopped by distraction (e. g., calling one's name)" (p. 1)7. Tales alteraciones no afectan la actividad motora del niño, aunque eventualmente pueden interrumpir su cotidianidad o provocar lesiones severas.

Asimismo, es posible distinguir entre estereotipias primarias y secundarias. Las primarias, asociadas a niños con desarrollo psicomotor normal, pueden estar presentes de forma temporal o crónica, mientras que las secundarias están asociadas a problemas de origen neurobiológico como el TEA. Un aspecto importante es que "the severity and frequency of motor stereotypies in ASD is correlated with severity of illness, degree of ID, and impairments in adaptive functioning and symbolic play" (Péter et al., 2017, p. 2) ${ }^{8}$. Las estereotipias motoras pueden presentarse de forma particular según cada individuo.

Por último, los niños diagnosticados con TEA se relacionan con los objetos de forma muy particular. Un aspecto notable es el interés que desarrollan por girar, ordenar, alinear y mover los juguetes u objetos de forma específica. Ante la lógica de cada niño, los objetos se organizan siguiendo un patrón repetitivo en donde es posible observar las características de cada uno de estos. La selección de los objetos está basada únicamente en sus características físicas; es decir, el niño muestra interés por estos, ya sea por su forma, color, olor o sabor. Así, el niño puede sentirse atraído por el color de una pelota o por la suavidad de un cojín, y suele presentar conductas de enojo o llanto cuando se le intenta privar de su proceso de exploración o del objeto.

Inflexibilidad de rutinas y patrones ritualizados de comportamiento verbal o no verbal

En el caso de los niños con TEA, se puede observar principalmente conductas ritualistas que se mantienen fijas en el tiempo y se ejecutan de manera habitual sin modificaciones o alteraciones. Estos niños mantienen una posición rígida y poco convencional. Rutter (1978, como se cita en López \& García, 2007) plantea cuatro conductas ritualistas (véase la figura 2).

7 “Las estereotipias motoras son movimientos repetitivos, rítmicos, a menudo bilaterales, con un patrón fijo (por ejemplo, aleteo, agitación o rotación de la mano) y una frecuencia regular que, por lo general, puede detenerse mediante una distracción (por ejemplo, llamar por su nombre)" [traducción de las autoras].

8 "La gravedad y la frecuencia de las estereotipias motoras en el TEA se correlacionan con la gravedad de la enfermedad, el grado de DI y las deficiencias en el funcionamiento adaptativo y el juego simbólico" [traducción de las autoras]. 
Figura 2

Conductas ritualistas presentes en el TEA

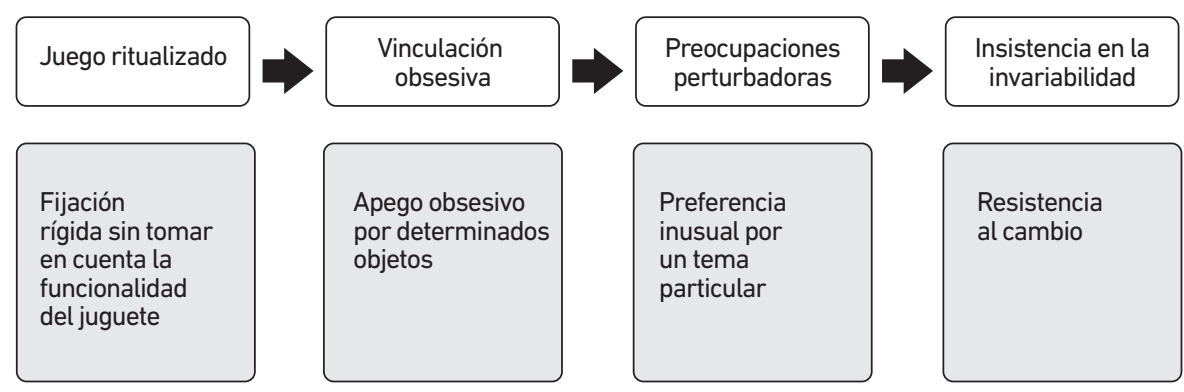

Fuente: Adaptado de Rutter (1978, como se cita en López \& García, 2007)

En cuanto al juego ritualizado, este es de carácter limitado y rígido. En este proceso, existe una fijación en las características de los objetos o juguetes más que en su valor simbólico o funcional. Se observa también la vinculación obsesiva con los objetos que posee, de manera que desarrolla una especie de apego con objetos particulares, negándose a dejarlos o cambiarlos. Estos objetos pueden ser desde un pedazo de cinta hasta un electrodoméstico. Un aspecto preocupante es el afán que adquieren estos niños por mantenerse próximos a estos objetos llegando incluso a dormir con ellos o estar durante todo el día deseándolos o pidiéndolos. Estas fijaciones están relacionadas con las estereotipias.

Hiper-o hiporreactividad a los estímulos sensoriales del entorno

Las personas diagnosticadas con TEA presentan experiencias sensoriales atípicas que influyen directamente en la forma en la que se relacionan con su entorno. En ese sentido, Friedlander (2009) indica que "sensory integration is another troublesome spot. Students with an ASD can have difficulty regulating input into their central nervous system, resulting in sensitivity to touch, sound, taste, or smell" (p. 141) ${ }^{9}$.

En el DSM-5 se plantean algunos ejemplos relacionados con las anomalías sensoriales, tales como la fascinación por las luces u objetos que giran; la respuesta a texturas, sonidos y olores específicos; la exploración por medio del tacto y del gusto de forma excesiva; la indiferencia al dolor o las temperaturas (American Psychiatric Association,

9 "La integración sensorial es otro punto problemático. Los estudiantes con TEA pueden tener dificultades para regular la entrada en su sistema nervioso central, lo que resulta en sensibilidad al tacto, sonido, gusto u olfato" [traducción de las autoras]. 
2013). Es preciso especificar que es posible que un mismo sujeto manifieste más de una anomalía sensorial. Estas anomalías son causadas por la hiperreactividad e hiporreactividad que estos niños presentan a los estímulos externos.

La hiperreactividad está relacionada con la hipersensibilidad o reacción sensorial alta a los estímulos. Esta puede generar, por un lado, que el niño se aísle del entorno para evitar relacionarse con estímulos que le generan displacer y, por otro lado, que reaccione con comportamientos agresivos y de oposición. La hiporreactividad es una reacción baja o pasiva ante los estímulos sensoriales, que puede suprimir de forma involuntaria diversas experiencias sensoriales. Esto llevará a una búsqueda activa de estímulos intensos que le permitan relacionarse y comprender su entorno.

Respecto a este apartado, Balasco et al. (2020) plantean deficiencias olfativas, gustativas, auditivas y visuales que se visibilizan en niños diagnosticados con TEA (véase la tabla 1).

Tabla 1

Deficiencias sensoriales en los niños con TEA

\begin{tabular}{ll}
\hline Tipo & Características \\
\hline Nivel olfativo & $\begin{array}{l}\text { Alteraciones en el olfato para detectar nuevos olores; sin embargo, los olores } \\
\text { típicos son recordados con facilidad. Existen problemas para identificar olores } \\
\text { agradables y desagradables. }\end{array}$ \\
& $\begin{array}{l}\text { Su alimentación es menor en comparación con el resto; existen problemas } \\
\text { Nivel gustativo } \\
\text { con la textura de los alimentos, por lo que terminan rechazándolos. Menor } \\
\text { pivel auditivo }\end{array}$ \\
& $\begin{array}{l}\text { Alteración ante sonidos peculiares que pueden causar diversas reacciones en } \\
\text { el niño. Los ruidos cotidianos pueden llegar a alterarlo. Preferencia por la voz } \\
\text { humana. }\end{array}$ \\
& Procesamiento visual centrado en los detalles. \\
\hline
\end{tabular}

Elaboración propia a partir de Balasco et al. (2020)

Por lo expuesto anteriormente, es preciso tomar en consideración que algunos estímulos sensoriales como la bulla, un grito o una luz muy intensa pueden alterar a los niños, llevándolos a reaccionar de formas particulares e inesperadas, influyendo en su comportamiento. 


\section{CONCLUSIONES}

Aquello que se comprende, actualmente, como trastorno del espectro autista (TEA) ha evolucionado a lo largo del tiempo pasando por diversas formas de conceptualización; inicialmente, se lo concebía como una enfermedad psiquiátrica hasta que devino en lo que ahora se explica como una condición relacionada con una alteración en el neurodesarrollo. El autismo fue estudiado, primero, desde un enfoque categorial; sin embargo, diversos autores observaron que este es un espectro que alberga un conjunto de afectaciones; por tanto, no se puede encasillar a los niños que poseen esta condición en etiquetas diagnósticas. Además, el TEA se presenta en cada niño de forma diferente, de modo que para ayudarlos a enfrentar los retos que les deparará la vida, se les debe ofrecer una variedad de apoyos en función de sus necesidades particulares. Por esta razón, es necesario que los profesionales conozcan los signos de alerta que se pueden visibilizar en estos niños, sobre todo durante la primera infancia, de manera que se consiga un diagnóstico temprano y sea posible intervenir con mejores pronósticos (Valdez, 2019).

El TEA ha sido abordado desde diversos planos: psicológicos, biológicos, terapéuticos y educativos a fin de comprender y entender su naturaleza. En un inicio, fue abordado desde la mirada de Bleuler, quien sustentó que el autismo estaba relacionado con la esquizofrenia (paradigma psiquiátrico). Posteriormente, Kanner utiliza el término para referirse a niños con problemas en el contacto afectivo, lo desliga de las enfermedades mentales y propone cuatro dimensiones afectadas dentro de él. Con el paso de los años, en la época de los cuarenta y sesenta se aborda el TEA desde una perspectiva psicoanalítica, en la que se responsabiliza a las madres por el trastorno desarrollado por el niño. Esta postura es retirada posteriormente al identificar el gran daño provocado en las familias. Más adelante, surge un aporte revelador en el campo, la tríada de Wing, en la que, desde una mirada educativa, se plantea la necesidad de ver el TEA desde un enfoque continuo más que categorial. Con este aporte se impulsaron los aprendizajes para niños con esta condición. En 1994, en el DSM-IV se plantean los trastornos generalizados del desarrollo a través de un enfoque categorial que retoma ideas de Wing y, finalmente, en el 2013, se publica el DSM-5, que entiende el autismo como un trastorno dentro del cual se puede reconocer un conjunto de afectaciones visibilizadas dentro de un espectro. Es posible denotar que el TEA está en constante evolución; por tanto, es necesario que los profesionales puedan reflexionar en torno al tema, de modo que se eviten las creencias erróneas o los prejuicios que distorsionan el actuar profesional en el proceso de atender a niños diagnosticados con esta condición, sobre todo en el campo educativo, en el que los docentes pueden movilizar creencias o prejuicios al respecto.

Existen signos de alerta que facilitan la identificación de niños con TEA planteados en el DSM-5 por la American Psychiatric Association (2013). Cada uno de estos signos 
fueron profundizados dentro del presente ensayo. El TEA presenta dos principales déficits, entre los que se encuentran (i) deficiencias en la comunicación e interacción social; y (ii) patrones del comportamiento, interés y actividades restringidas y repetitivas. En el primero se puede visibilizar el déficit en la reciprocidad emocional, en las conductas comunicativas no verbales utilizadas en la interacción social y en el desarrollo y mantenimiento de las relaciones sociales. Con relación al segundo, se observa lenguaje, movimiento y utilización de objetos estereotipados y repetitivos; inflexibilidad en las rutinas y patrones ritualizados de comportamientos verbal y no verbal; intereses fijos y restringidos; e hiper-e hiporreactividad.

Es importante que los docentes conozcan sobre el TEA, ya que posibilita la reflexión activa en torno a las creencias o prejuicios que pueden tener sobre el trastorno, los cuales pueden estar interfiriendo en la práctica educativa. Este aspecto también se relaciona con la inclusión y las reales oportunidades que los niños con TEA tienen para acceder a una educación de calidad. El conocimiento de esta condición admitirá nuevas visiones y miradas en torno al tema, dejando a un costado conceptos o interpretaciones desactualizadas o prejuiciosas. Asimismo, permitirá comprender cómo se presentan las características o signos de alarma en los niños, lo que facilitará la aplicación de nuevas estrategias de apoyo para favorecer la inclusión educativa desde la educación infantil, lo más temprano posible.

El conocimiento del TEA es un punto de partida para garantizar una adecuada inclusión que atienda a los niños que presentan esta condición, ya que admite la posibilidad de desarrollar estrategias, según las necesidades de los estudiantes, con el propósito de favorecer mejores aprendizajes. Sería conveniente promover investigaciones que permitan indagar en las creencias docentes sobre el TEA, los primeros años de escolaridad de estos niños y sus aprendizajes, así como el trabajo con las familias. Se considera fundamental que las personas involucradas en el trabajo con estos niños puedan conocer más información acerca de este trastorno, la cual constituya un referente primordial para seguir investigando sobre este tema cuando se desarrolla en el campo educativo.

\section{REFERENCIAS}

Alayza, M. (2020). El método de revisión de la literatura. En A. O. Sánchez Huarcaya (Coord.), Los métodos de investigación para la elaboración de las tesis de maestría en educación (pp. 23-33). Pontificia Universidad Católica del Perú, Facultad de Educación.

American Psychiatric Association. (1995). Manual diagnóstico y estadístico de los trastornos mentales DSM-IV-TR. Masson. 
American Psychiatric Association. (2013). Diagnostic and Statistical Manual of Mental Disorders (5. ${ }^{\mathrm{a}}$ ed.).

Artigas-Pallares, J., \& Paula, I. (2012). El autismo 70 años después de Leo Kanner y Hans Asperger. Revista de la Asociación Española de Neuropsiquiatría, 32(115), 567-587. https://dx.doi.org/10.4321/S0211-57352012000300008

Ávila, H. (2006). Introducción a la metodología de la investigación. Edición electrónica. https://www.eumed.net/libros-gratis/2006c/203/

Balasco, L., Provenzano, G., \& Bozzi, Y. (2020). Sensory Abnormalities in Autism Spectrum Disorders: A Focus on the Tactile Domain, from Genetic Mouse Models to the Clinic. Frontiers in Psychiatry, 10, 1016. https://doi.org/10.3389/fpsyt.2019.01016

Bleuler, M., y Bleuler, R. (1986). Dementia praecox oder die Gruppe der Schizophrenien: Eugen Bleuler. The British Journal of Psychiatry, 149, 661-662. https://doi. org/10.1192/bjp.149.5.661

Booth, T., \& Ainscow, M. (2000). Índice de inclusión. Desarrollando el aprendizaje y la participación en las escuelas. UNESCO. http://www.daemcopiapo.cl/Biblioteca/ Archivos/INDICE_INCLUSION.pdf

Bruinsma, Y., Koegel, R., \& Kern, L. (2004). Joint Attention and Children with Autism: A Review of the Literature. Mental Retardation and Developmental Disabilities Research Reviews, 10, 169-175. https://doi.org/10.1002/mrdd.20036

Busquets, L., Miralbell, J., Muñoz, P., Muriel, N., Español, N., Viloca, L., \& Mestres, M. (2018). Detección precoz del trastorno del espectro autista durante el primer año de vida en la consulta pediátrica. Pediatría Integral, 22(2), 105.e1-105.e6. https://www. pediatriaintegral.es/publicacion-2018-03/deteccion-precoz-del-trastorno-delespectro-autista-durante-el-primer-ano-de-vida-en-la-consulta-pediatrica/

Cueto, S., Rojas, V., Dammert, M., \& Felipe, C. (2018). Cobertura, oportunidades y percepciones sobre la educación inclusiva en el Perú. Grupo de Análisis para el Desarrollo (GRADE). https://www.grade.org.pe/publicaciones/cobertura-oportunidades-ypercepciones-sobre-la-educacion-inclusiva-en-el-peru/

Decreto Supremo 011-2012-ED [Ministerio de Educación]. Aprueba Reglamento de la Ley General de Educación. 6 de julio del 2012. http://www.minedu.gob.pe/ files/3926_201207101510.pdf

Diazgranados, N., \& Tebar, M. (2019). La educación de las personas con trastorno del espectro autista: Colombia en los últimos 10 años. Horizontes Pedagógicos, 21(1), 1-10. https://doi.org/10.33881/0123-8264.hop.21101 
Friedlander, D. (2009). Sam Comes to School: Including Students with Autism in Your Classroom. The Clearing House, 82(3), 141-144.

Garrabé, J. (2012). El autismo: historia y clasificaciones. Salud Mental, 35(3), 257-261. http://www.scielo.org.mx/scielo.php?script=sci_arttext\&pid=S0185$33252012000300010 \& \operatorname{lng}=e s \&$ tlng $=e s$

Ghanizadeh, A. (2010). Clinical Approach to Motor Stereotypies in Autistic Children. Iranian Journal of Pediatrics, 20(2), 149-159.

Gómez, L. (2011). Un espacio para la investigación documental. Revista Vanguardia Psicológica Clínica Teórica y Práctica, 1(2), 226-233.

González, M. (2015). Trastorno del espectro autista: una perspectiva pedagógica dirigida al profesorado. [Tesis de maestría, Universidad de Cantabria]. https://repositorio. unican.es/xmlui/bitstream/handle/10902/7777/GonzalezOrtizMarinaJosefa. pdf?sequence $=1$ \&isAllowed $=y$

Lam, L. (2014). Symbolic Play in Children with Autism. En V. Patel, V. Preedy \& C. Martin (Eds.), Comprehensive Guide to Autism. Springer. https://doi.org/10.1007/978-14614-4788-7_26

Ley 28044 del 2003. Ley General de Educación. 28 de julio del 2003. http://www.minedu. gob.pe/p/ley_general_de_educacion_28044.pdf

Ley 30150 del 2014. Ley de Protección de las Personas con Trastorno del Espectro Autista. 8 de enero del 2014. https://busquedas.elperuano.pe/ normaslegales/ley-de-proteccion-de-las-personas-con-trastorno-del-delespe-ley-n-30150-1035199-2/

López, S., \& García, C. (2007). Patrones comportamentales en el trastorno autista: descripción e intervención psicoeducativa. Psicología Educativa, 13(2), 117-131. https://journals.copmadrid.org/psed/archivos/105082.pdf

Manrique, S., del Castillo, M., Valdez, D., Garrido, G., Rattazzi, A., Paula, C., García, R., Rosoli, A., Cukier, S., \& Montiel-Nava. (2019). Necesidades y calidad de vida de familias de personas con trastorno del espectro autista (TEA) en Perú. Implementación de la encuesta de la Red del Espectro Autista Latinoamérica (REAL). En D. Valdez (Ed.), Autismo. Claves para la intervención psicoeducativa. Ediciones Libro Amigo.

Meneses Montero, M., \& Monge Alvarado, M. Á. (2001). El juego en los niños: enfoque teórico. Revista Educación, 25(2),113-124.

Mergl, M., \& Salgado, C. (2015). Echolalia's Types in Children with Autism Spectrum Disorder. Revista CEFAC, 17(6), 2072-2080. https://doi.org/10.1590/1982021620151763015 
Miguel, A. (2006). El mundo de las emociones en los autistas. Teoría de la Educación. Educación y Cultura en la Sociedad de la Información, 7(2), 169-183. https://www. redalyc.org/articulo.oa?id=2010/201017296011

Ministerio de Educación. (2012). Reglamento de la Ley General de Educación N.² 28044. http:// www.minedu.gob.pe/normatividad/reglamentos/EducacionCalidadyEquidad. pdf

Ministerio de la Mujer y Poblaciones Vulnerables, \& Consejo Nacional para la Integración de la Persona con Discapacidad. (2019). Plan Nacional para las Personas con Trastorno del Espectro Autista. https://www.gob.pe/institucion/conadis/ informes-publicaciones/265413-plan-nacional-para-las-personas-con-trastorno-del-espectro-autista-2019-2021

Morueco Alonso, M. (2015). Categorías de enseñanza-aprendizaje para alumnos del trastorno del espectro autista [Tesis doctoral, Universitat de les Illes Balears]. http:// hdl.handle.net/11201/148780

Organización Mundial de la Salud. (2013). Medidas integrales y coordinadas para gestionar el trastorno del espectro autista. Informe de la Secretaría EB133/4. https://apps. who.int/iris/bitstream/handle/10665/172324/B133_4-sp.pdf

Peretti, L. (2010). La reciprocidad socio-emocional como una capacidad gradual en personas con autismo. En P. García \& A. Massolo (Eds.), Epistemología e historia de la ciencia. Selección de trabajos de las XX Jornadas (vol. 16, pp. 478-487). Universidad Nacional de Córdova. https://rdu.unc.edu.ar/handle/11086/3532

Péter, Z., Oliphant, M., \& Fernandez, T. (2017). Motor Stereotypies: A Pathophysiological Review. Frontiers in Neuroscience, 11(171), 1-6. https://doi.org/10.3389/ fnins.2017.00171

Piaget, J. (1991). Seis estudios de psicología. Editorial Labor. http://dinterrondonia2010. pbworks.com/f/Jean_Piaget__Seis_estudios_de_Psicologia.pdf

Pontificia Universidad Católica del Perú. (2016). Reglamento del Comité de Ética de la Investigación de la Pontificia Universidad Católica del Perú. https://departamento. pucp.edu.pe/psicologia/wp-content/uploads/2019/08/reglamentodelcomitedeeticadelainvestigaciondelapontificiauniversidadcatolicadelperu.pdf

Reynoso, C., Rangel, M., \& Melgar, V. (2015). El trastorno del espectro autista: aspectos etiológicos, diagnósticos y terapéuticos. Revista Médica del Instituto Mexicano del Seguro Social, 55(2), 214-22.

Valdez, D. (2005). Teoría de la mente, memoria autobiográfica y síndrome de Asperger. Fundamentos para la intervención clínica y educativa. Revista El Cisne, 15(179), 1-8. 
Valdez, D. (2012). Desarrollo comunicativo. En J. Castorina \& M. Carretero (Comps.). Desarrollo cognitivo y educación. Los inicios del conocimiento (pp. 196-217). Paidós.

Valdez, D. (2019). Autismo: cómo crear contextos amigables. Paidós.

Wang, X., Desalvo, N., Zhao, X., Feng, T., Loveland, K., Shi, W., \& Gnawali, O. (2014). Eye Contact Reminder System for People with Autism. En 6th International Conference on Mobile Computing, Applications and Services (pp. 160-163). https://doi: 10.4108/ icst.mobicase.2014.257796

Wing, L., \& Gould, J. (1979). Severe Impairments of Social Interaction and Associated Abnormalities in Children: Epidemiology and Classification. Journal of Autism and Developmental Disorders, 9, 11-29. https://doi.org/10.1007/BF01531288 